\title{
La magie du temps et des saisons dans la dramaturgie de Paul Willems
}

\author{
The magic of weather and seasons \\ in the theater of Paul Willems
}

\author{
Renata Jakubczuk \\ Uniwersytet Marii-Curie Skłodowskiej \\ renata.jakubczuk@umcs.pl
}

\begin{abstract}
In the current article we are examining the presence, importance and significance of weather and seasons in dramatic works of the Belgian writer, Paul Willems. Our study relies upon the methodology of patternsof transitivity proposed by Jean-Pierre Richard. After explaining the principals of our approach, then presenting the corpus of the studied texts and formulating our basic hypothesis concerning the weather, we are reviewing four weather phenomena: the rain, the wind, the snow, and the sun. The completed analysis confirmed the calming role of the rain, the soothing role of the sun and the warning role of the wind. However, we realized, besides the liberating role of the snow in the introduction, its more complex significance because it "weighs heavily" on the life of the characters.
\end{abstract}

Keywords: Paul Willems, theater, rain, snow, transitivity scheme

J'avais appris à célébrer l'instant comme on célèbre une messe. Mes professeurs étaient le vent, la pluie, la lumière, la neige, le gel,

l'eau de l'étang, l'herbe ou les feuilles

(Willems, 1998, p. 44).

Où sont les oiseaux [...] Au fond des saisons

(Willems, 2018, p. 62). 


\section{INTRODUCTION}

Si l'œuvre narrative de Paul Willems est davantage commentée par les critiques littéraires, ses pièces de théâtre n'ont pas encore été sujettes à une étude exhaustive ${ }^{1}$. De plus, dans les archives demeurent encore des textes inédits de ce « dernier écrivain belge » (Loriau, Pantkowska, 2001). Or son écriture dramatique s'avère être une production originale qui diffère largement des tendances de son époque ${ }^{2}$ par, ce que les critiques appellent, le « réalisme magique » de Willems ${ }^{3}$.

Né en 1912 à Edegem, près d'Anvers en Flandre belge, Paul Willems grandit dans la demeure familiale de Missembourg ; « un lieu protégé, un monde isolé du Monde, où seuls les saisons et les météores avaient libre accès » (Willems, 1975, p. 326); entourée d'un étang (disparu dans les années 30) et d'un terrain boisé de châtaigniers triséculaires formant ainsi une île ${ }^{4}$; île magique pour l'enfant qui y vit, joue et apprend puisqu'il ne fréquente l'école qu'à partir du secondaire, à l'âge de douze ans. De même que sa mère, Marie Gevers (1883-1975), le fils est séduit par la beauté du domaine $^{5}$ qui fut une source d'inspiration inappréciable pour les deux écrivains ${ }^{6}$. De plus, la proximité de la mer et surtout du fleuve, l'Escaut - près d'Anvers, il est immense - jouent un rôle très important aussi bien dans la vie que dans l'œuvre de Paul

${ }^{1}$ Il est nécessaire de citer néanmoins une monographie de Rosalba Gasparro Oltre il giardino. Paul Willems tra narrativa e teatro, parue en 2014 chez Bulzonie à Rome, qui englobe l'ensemble de l'œuvre de l'écrivain belge.

${ }^{2}$ Les pièces de Paul Willems datent de la seconde moitié du XXe siècle. Rappelons que c'est la période d'un grand bouleversement dans le théâtre européen lié au théâtre de l'absurde en France. Mais c'est aussi l'époque de l'existentialisme avec le théâtre à thèse de Sartre ou celui un peu fantastique et drôle d'Anouilh en France. Soulignons d'emblée que l'absurde n'a pas tellement marqué la Belgique où on note le théâtre « humain » de Louvet ou le théâtre inclassable de Kalisky ou l'influence de la littérature anglo-saxonne postbrechtienne (Thomas Bernhard, Heiner Muller). La création poétique de Paul Willems reste une création à part.

${ }^{3}$ Le phénomène a été largement décrit par Marc Quaghebeur dans son excellent texte « Du fantastique réel au réalisme magique ou d'une guerre mondiale à l'autre. Tout est réel ici de Paul Willems » (Lublin 2017).

${ }^{4}$ Grâce à un documentaire réalisé en 2014 par Marc Quaghebeur et les Archives et Musée de la Littérature de Bruxelles, on peut découvrir et admirer la beauté de la résidence familiale.

${ }^{5}$ Il convient d'ajouter que c'est Florent Gevers, le père de Marie, qui acheta Missembourg (à l'époque appelé Mussenborg) au milieu du XIX ${ }^{\mathrm{e}}$ siècle. Il l'aima tellement qu'il renonça à son voyage de noces à Venise... Quant à Marie, son premier recueil de poèmes porte le titre très significatif : Missembourg (1918). Un siècle et demi plus tard, le fils de Paul Willems - Jan - vend la propriété familiale dont l'entretien coûte trop cher. Il quittera le domaine fin octobre 2020.

${ }^{6}$ «L'œuvre de Marie Gevers est liée si étroitement à Missembourg qu'il y a une véritable osmose entre elle et la vieille maison, le jardin, les arbres, la pluie, les roses, les oiseaux, le brouillard, les bourgeons, la neige, les quatre vents du ciel ainsi que les parfums. J'oubliais : l'étang ! Il est vide. Pourtant il vit toujours dans notre mémoire et nous portons en nous ses reflets. Quoiqu'il n'y ait plus que de larges douves vides et des étendues vaseuses, l'étang » nous offre, portés par le souvenir, les ciels révolus et l'eau disparue ", écrit Paul Willems sur la création de sa mère (Willems, 1989, p. 5). Pourtant, ces caractéristiques sont valables aussi pour l'œuvre du fils... 
Willems. L'auteur y revient aussi bien dans ses romans (Tout est réel ici) que dans sa production dramatique (La Ville à voile). D'autre part, on remarque aussi la présence de l'île autour de laquelle est nouée toute l'action de Warna ou le poids de la neige ou d'Elle disait dormir pour mourir. Si l'on n'oublie pas que l'écriture de Paul Willems s'inscrit dans l'héritage du symbolisme belge (Crommelynck, Maeterlinck), on comprend mieux cette imprégnation foncière qui caractérise son œuvre.

Or la vie proche de la nature est forcément influencée par le temps et les saisons auxquels est finalement soumise toute l'activité, toute la vie humaine ${ }^{7}$. L'incipit d'un texte de Willems, consacré à sa mère, Marie Gevers, reste bien significatif à ce sujet : « J'écris ces lignes dans le jardin, par un jour de septembre. Là-bas, une rangée de peupliers du Canada tremble dans la lumière. Bruissement se gonflant et s'apaisant. La musique des feuilles est une des voix secrètes du monde » (Willems, 1975, p. 324).

L'article que nous proposons, et qui se voudrait une contribution à la fresque willemsienne qui fait toujours défaut, se focalisera sur un certain nombre de procédés mis en œuvre par l'auteur pour souligner l'importance du temps et des saisons dans la vie humaine : l'apaisement de la pluie qui alimente les sols souillés ou épuisés et offre une douche de propreté ; la délivrance de la neige qui couvre par une nappe blanche et innocente toutes les saletés et les impuretés, mais aussi les secrets ou les vérités repoussées de la vie terrestre ; le soulagement du soleil qui redonne à l'homme de l'optimisme et des forces vitales ; l'avertissement du vent qui pénètre profondément et finit par purifier l'air et l'ambiance... Soulignons d'emblée que la violence des éléments nous rappelle notre place dans l'univers dès l'origine, mais, en même temps, nous en rend totalement dépendants. L'homme y est impuissant et ne peut que solliciter ou attendre leur venue car rien ne dépend de lui. D'autre part, il ne faut pas oublier que leur rôle est toujours double : soit salvateur soit destructeur. Cette dichotomie est fondamentale car la frontière entre les deux est souvent très fine, surtout par rapport à l'eau. Si l'omniprésence de l'élément aquatique dans l'œuvre de Paul Willems ne suscite pas de controverses - les chercheurs en soulignent l'importance dans plusieurs publications $^{8}$ - la neige 9 , le soleil, le vent, le passage d'une saison à une autre, ne sont pas tellement observés dans les travaux des chercheurs.

\footnotetext{
${ }^{7}$ « Les défenses les plus sûres contre le chagrin et le mal sont les enceintes d'arbres et les barrières des étangs. Les reflets de l'eau nous protègent mieux que les pont-levis ou les fusées antifusées. Pour se protéger mieux encore mon grand-père avait inventé son autarcie. Le jardin potager et le verger lui donnaient fruits et légumes en toutes saisons. Les dernières pommes se mangeaient en mai au moment où rougissaient les premières cerises, et l'on croquait toute l'année des noisettes, dont les dix variétés rouges, vertes, blanches, rondes ou ovales avaient chacune leur goût et leur mois d'excellence. On n'achetait que le sel et la viande. Le pain était cuit dans la petite ferme du jardin. [...] On vivait en un cycle harmonieusement clos » (Willems, 1975, p. 327).

${ }^{8}$ Cf. Barbara Wright $(1984,1992)$, Éric Lysøe (2002), Renata Jakubczuk $(2018,2019)$ ou le numéro entier de la revue Lublin Studies in Modern Languages and Literature (42 (3), 2018).

${ }^{9}$ Sauf l'article de Marie Giraud-Claude-Lafontaine (2018) «La neige et la guerre dans La neige était sale de Georges Simenon et Warna ou le poids de la neige de Paul Willems ».
} 
Pour ce qui est de l'approche méthodologique, nous proposons de nous appuyer sur la stratégie/technique/démarche de l'analyse littéraire proposée par Jean-Pierre Richard dans Onze études sur la poésie moderne. Conçue pour des œuvres lyriques - l'auteur y étudie la poésie de onze poètes de la seconde moitié du XX $\mathrm{X}^{\mathrm{e}}$ siècle - elle nous semble pertinente pour être appliquée au « théâtre poétique ${ }^{10}$ de Paul Willems.

En tentant d'aborder la création littéraire d'un auteur dans son ensemble, JeanPierre Richard propose d'y retrouver des similitudes qui permettraient de relier les œuvres successives entre elles, mais aussi de repérer des connecteurs thématiques ou des liens isotopiques à l'intérieur d'une œuvre. Il les nomme des « schèmes ». Néanmoins, ces schèmes peuvent se manifester et fonctionner de façons différentes : « de juxtaposition, de mélange, d'organisation, d'ambiguïté, de métamorphose » (p. 8), « de transitivité » (p. 246) ou «d'incision » (p. 295). Jean-Pierre Richard explique aussi que « les structures verbales, qui informent l'ordre de l'horizontalité signifiante [...] y entrent en rapport d'homologie avec les structures de perception ou de rêverie, telles qu'elles se disposent, en grappes, dans la latence du poème - dans l'ordre de la verticalité métaphorique, de la sélection. C'est sans doute cet isomorphisme qui distingue de toutes les autres la signification proprement littéraire » (p. 10).

Il n'est pas anodin de rappeler que Paul Willems parle des unités au théâtre, mais pas dans le sens orthodoxe attribué à ces termes. Les unités évoquées par le dramaturge répondraient plutôt aux schèmes de transitivité proposées par Richard. Reprenons l'explication de l'écrivain belge :

Le langage des personnages, leurs réactions psychologiques et leur comportement doivent puiser aux sources de l'inconscient mais ne jamais être gratuits ; au contraire, à leur façon, ils doivent être implacablement logiques, mais de la logique du rêve, qui est métaphorique et analogique, c'est-à-dire qu'elle vient du monde perçu par leur sens. Pour que la pièce soit perçue par le public, il faut qu'il y ait unité de métaphore ${ }^{11}$. En d'autres mots, toutes les images utilisées dans une pièce doivent être de la même famille, de la même palette ${ }^{12}$. C'est cela qui fera l'unité de vision. [...] Au théâtre, ce qui commence par un arbre, la pluie et un client qui traverse les murailles doit se terminer par un arbre, la pluie et un passe-muraille. Dans Il pleut dans ma maison, j'ai veillé à ne pas trahir l'unité de l'image. [...] L'unité de l'image demande l'unité du vocabulaire. Or, le comportement des mots est étrange et mystérieux. On n'a jamais, je crois, élucidé par quelles voies ils atteignent la conscience (Willems, 1998, p. 90-91).

Afin de mieux cerner l'originalité de l'approche willemsienne, nous envisageons d'étudier - à côté des deux pièces emblématiques pour notre sujet : Il pleut dans ma

${ }^{10}$ Dans un des premiers textes consacrés au théâtre (1957), Paul Willems écrit : « Le théâtre est le lieu, par excellence, où s'épanouit l'irrationnel. Violence ou douceur, foi ou doute, tragique ou comique, la vie elle-même est transposée sur la scène et parle le langage de la poésie »; le texte suivant, de 1958, est intitulé À propos du théâtre poétique (Willems, 2004, p. 20, 23).

${ }^{11}$ C'est nous qui soulignons. De même que les autres formes des unités proposées par le dramaturge.

${ }^{12}$ C'est l'auteur qui souligne. 
maison (1962) et Warna ou le poids de la neige (1963) ${ }^{13}$ - également d'autres textes de l'écrivain belge dans lesquels nous allons rechercher l'influence et la puissance des éléments naturels : La Ville à voile, La Vita breve, Off et la lune, La Plage aux anguilles, Elle disait dormir pour mourir.

\section{PAR TEMPS DE PLUIE}

Il n'est pas étonnant que, dans le plat pays qu'est la Flandre, c'est la pluie qui joue le premier violon. Dans plusieurs textes du dramaturge, on peut trouver des descriptions poétiques de la pluie. À titre d'exemple, citons le fragment suivant :

La nuit. Je dérivais dans le bruissement de la pluie. Près de moi, dans la chambre, j'entendais tomber les gouttes, musique, carillon des fées. Les unes tombaient sur le tapis avec un son étouffé, les autres sur le plancher, et c'était un son mat, sur la cheminée de marbre, un son net et sur la table de toilette, dans le bassin de porcelaine, un son vif; et tout autour de la maison, sans désemparer, la pluie jouait dans les arbres. C'était la musique du hasard. Berceuse exquise (Willems, 1998, p. 89).

Régi par les lois de la scène, un texte dramatique est censé emprunter d'autres moyens d'expression pour rendre une telle image. Voici la réplique que le dramaturge met dans la bouche de Bulle, l'un des personnages d'Il pleut dans ma maison :

[...] une partie de la pluie tombe dans le jardin, et l'autre sur le toit. La partie qui tombe sur le toit se divise en deux, l'une qui dégouline dans la gouttière, et l'autre qui... S'introduit [...]. La partie qui s'introduit dans la maison se divise en quatre. La première reste au grenier où elle s'ennuie, la seconde va au premier étage dans les lits, où elle s'amuse ; la troisième descend l'escalier par petites cascades, et la quatrième, et la quatrième s'infiltre ici, où elle arrose l'arbre, goutte à goutte (Willems, 2018, p. 97) ${ }^{14}$.

Tout aussi poétique que la précédente, cette description renvoie à l'espace central de la pièce, Grand'Rosière, « une vieille maison de campagne en ruines » (PMM, p. 75) avec un arbre qui pousse au milieu du salon. Pour les habitants de la maison, cet arbre « est très beau. Il tient toute la maison dans ses branches » (PMM, p. 75). Pour les étrangers, il l'abime en soulevant le parquet, en trouant le plafond, en défonçant les fenêtres et laissant entrer la pluie à l'intérieur... Rosalba Gasparro y voit une « métaphore paradisiaque de l'arbre, qui s'enracine et protège les personnages [...]» (2002, p. 232).

${ }^{13}$ Initialement publié en 1963, puis avec des variantes dans la collection Didascalies (1984) avec une Lettre de l'auteur aux acteurs. Nous nous appuyons sur l'édition récente parue en 2018 dans les Archives du Futur.

${ }^{14}$ Afin de rendre la lecture plus facile, dorénavant, nous allons utiliser le sigle $P M M$, suivi de la pagination et mis entre parenthèses. 
En exergue de la pièce, le dramaturge a mis un poème intitulé Il pleut. Lors de la première représentation, en 1962 au Rideau de Bruxelles, il a distribué au public des feuilles de platane avec le poème suivant :

\section{Rêvons !}

Il pleut.

Nos destins sont fermés

Les rêves et les reflets

Sont nos seuls liberté ${ }^{15}$.

En effet, si la pluie n'apparaît qu'aux moments-clés de l'intrigue, les rêves et les reflets accompagnent les dramatis personnce tout au long de l'action ${ }^{16}$. Au lever du rideau, Madeleine, propriétaire de la maison, s'en vient pour la vendre. Mais ses habitants y sont heureux. Ils font tout pour empêcher la transaction. Dès l'incipit, Madeleine se place en opposition à tout le monde et ne comprend rien de la façon de vivre « dans un monde arrêté il y a cinquante ans... [...] dans le Temps Immobile » (PMM, p. 88). Tout au long du premier acte, les deux « camps adversaires » agissent pour faire tirer plus leurs causes et cela jusqu'à l'arrivée de la pluie. La dichotomie entre les attitudes des « autochtones » et de l'« étrangère » s'y manifeste nettement car « ils tendent tous la main, sauf MADELEINE » peut-on lire dans les didascalies. Pour les habitants de Grand'Rosière, qui vivent en parfaite harmonie avec la nature, la pluie est un phénomène naturel. Bulle la perçoit comme une délivrance : « Ce matin, le ciel était couvert. Avant de me réveiller, je le devinais. J'avais la sensation d'un plafond. J'ai pensé, tiens, de la pluie pour ce soir » (PMM, p. 97).

Le deuxième acte se termine par l'annonce d'une catastrophe : « Ils vont vendre Grand-Rosière » (PMM, p. 131), ce qui arrivera d'ailleurs. Mais le troisième acte débute avec la pluie : «Les bassines sont disposées partout comme à la fin du premier acte. On entend la pluie. Il [Bulle] tend la main pour recevoir des gouttes de pluie » (PMM, p. 132). Elle accompagnera les personnages jusqu'à la nuit et ne s'arrêtera qu'à l'aube. À en croire Christophe Meurée : «L'unité de palette de la pièce tient en effet à l'alliance des trois couleurs que sont l'arbre, la pluie et la porosité entre les mondes d'ici et de là-bas. La présence de l'arbre favorise l'intrusion de la pluie au sein de la maison jusqu'à rendre perméables les frontières entre les mondes et autoriser toutes les logiques de passe-muraille» $(2018$, p. 48).

${ }^{15}$ Une reproduction de cette feuille se trouve dans l'édition de 1962. Elle est également reprise - en couleurs - dans celle de 2018.

${ }^{16}$ Bien que les termes « intrigue » et « action » soient souvent employés de façon synonymique, nous préférons leurs significations plus précises : «L'action se situe à un niveau relativement profond [...] [et] peut être résumée en un code général et abstrait » et « L'intrigue, par opposition à l'action, est la suite détaillée des rebondissements de la fable, l'entrelacement et la série des conflits et des obstacles et des moyens mis en œuvre par les personnages pour les surmonter» (Pavis, 2009, p. 8 et p. 179). 


\section{PAR TEMPS DE VENT}

À l'instar de la pluie, le vent est largement présent dans les écrits de Paul Willems :

C'est ma mère qui m'a initié au vent. Par exemple, elle m'a appris la qualité de l'air à l'ouïe. Le matin tôt, quand le vent apportait l'appel des cloches de l'église d'Edegem, sans ouvrir les yeux, rien qu'à entendre le son humide et enveloppé et souple de la cloche je savais que le vent avait tourné à l'ouest, que les nuages volaient bas et que la pluie viendrait. Mais pas n'importe quelle pluie : une longue, douce et fine pluie. Un grand bien-être m'envahissait.

En revanche, quand j'entends en hiver les cloches de l'église de Hove, je sais aussitôt que le vent est à l'est. Le son est métallique, et clair et sec et froid. Je sais que je ne dormirai plus, que ma pensée aura des ailes, et que - joie ! - je puis préparer mes patins qui dorment dans l'armoire.

D'autres fois, quand j'entends par bouffées les vagues rumeurs du port d'Anvers, le vent est au nord, je subodore les giboulées, et j'ai envie de m'embarquer pour un long voyage vers une contrée froide (Willems, 1998, p. 15).

Pour ce qui est de son œuvre dramatique, la pièce qui s'impose au sujet du vent est La Ville à voile dont le titre même évoque la présence et l'importance du royaume d'Éole ${ }^{17}$. L'intrigue d'un autre texte paru pour la première fois dans l'édition de poche de la collection Espace-Nord, La Vita breve ${ }^{18}$, se passe entièrement sur un bateau, espace particulièrement propice à l'intervention des vents.

Les deux drames s'ouvrent sur l'évocation du vent : « Le vent se lèvera tout à l'heure. L'eau du dock pressent le vent d'ouest » (VV, p. 22) dit Anne-Marie en répondant à côté de la question que son père lui a posée. Il en est de même dans le deuxième acte où elle dit « On entend le vent. [...] Une tempête. Je l'avais prévue. Une tempête sur l'Escaut » $(V V$, p. 65,66$)$; ainsi que dans le troisième où les didascalies précisent que l'on entend le vent (p. 102). La transformation du vent « simple» en tempête intervient comme une annonce de quelque chose de grave comme si la nature voulait de façon quasi magique avertir les personnages - dont Anne-Marie en premier - de l'événement qui adviendra ${ }^{19}$. Cette fonction du vent est confirmée dans Warna où la protagoniste éponyme demande : «Visius, y a-t-il du vent cette nuit ? » et ce dernier

${ }^{17}$ L'auteur l'explique ainsi : « Il y a d'abord sa construction en miroir : La Ville / [l]à voile. [...] Le $\mathrm{V}$ est aussi la première lettre de vent, et évoque phonétiquement le vent (comme le wind flamand, anglais et allemand). Le $\mathrm{V}$ convient aussi à voile, épouse du vent, et à Anvers, sa maîtresse qui l'attend d'où qu'il vienne : du nord, du sud, de l'est ou de l'ouest » (Willems, 1998, p. 110).

${ }^{18}$ Les citations venant de ce volume seront abrégées en sigles $V V$ pour La Ville à voile et $V B$ pour La Vita breve, suivis de la pagination.

${ }^{19}$ Dans les didascalies du deuxième acte, l'auteur précise : « Soudain, entrent les bruits du grand vent sur la ville. Pêle-mêle, cloches, volets, rafales de pluie, moteurs, feuilles et papiers qui glissent le long des murs, couvercles de poubelles, cordages détachés de la maison à voile battant régulièrement sur le toit, parfois une bouffée de musique aussitôt emportée, des pas qui courent. Il faut que cette tempête soit autre 
répond : «Non, Madame, tout est immobile. Immobile comme si quelque chose allait se passer » (WPN, p. 303).

Ce qui peut surprendre dans La Ville à voile, c'est le fait qu'après la tempête, le vent disparaît jusqu'à la fin de la pièce en cédant la place au soleil, présent au quatrième acte dans la seconde version. Nous avons constaté aussi qu'à côté d'Anne-Marie, seul Josty (p. 48 et 69) et Dile (p. 83,91) évoquent le vent avec une certaine nostalgie. Ce dernier divise le vent en celui de l'est, par un jour de février le moment de sa rencontre avec Anne-Marie, et celui de l'ouest ${ }^{20}$ qui emmène la tempête de novembre.

Dans La Vita breve, une voix mystérieuse chante de façon magnifique. Premièrement, on entend les mots séparés qui prennent la forme d'un poème chanté au quatrième épisode :

Le vent mêlé au temps!

Lamer!

Lamer!

Que de cris dans le vent!

Le temps!

Le temps!

Le vent amer (VB, p. 179).

Ils réapparaissent en excipit de la pièce sous forme d'un autre poème, parlé par Hamalissa :

$[\ldots]$ Le ciel a peur du temps. [...]

Que de cris sur la mer.

Le vent

Le vent

Le vent mêlé au temps (p. 259).

La boucle ainsi créée par le dramaturge met en relief l'importance de cet élément naturel. Les indications venteuses interviennent aussi dans d'autres textes, même si le vent n'y revêt pas une même signification. Citons quelques exemples : en parlant d'une auberge de luxe, Germaine évoque « des fenêtres défoncées pour entendre le vent » (PMM, p. 78) et Bulle n'est nullement surpris par l'arrivée de la pluie car « le vent est à l'ouest depuis hier. À l'ouest» (PMM, p. 96). Même M. Doré, qui ne raisonne qu'à trouver l'argent, se réfère aux éléments naturels : «Et l'occasion est comme la pluie et le beau temps, elle vient avec le vent » (PMM, p. 134).

chose qu'une tempête de théâtre. Elle devrait être composée comme la musique de la nuit. On entend toute la ville et ses épaves qui dérivent $»(V V$, pp. 77-78).

${ }^{20}$ Marc Quaghebeur parle même de « l'écriture du vent d'ouest » dans la création willemsienne (1992, p. 309). 
Une autre appréhension du vent est perceptible dans Warna où le temps hivernal amène le vent glacial qui fixe le statu quo dramatique pendant très longtemps. Le vent humide est « souple comme un chat» (WPN, p. 245). Cette personnification de la nature n'est pas gratuite car c'est le vent qui empêche les changements. Le dénouement n'est possible qu'avec l'aide des forces de la nature : « Le vent a changé ! Les nuages viennent du sud! Demain, le dégel! À la première goutte de pluie, je pleurerai de joie. Le printemps! » (WPN, p. 304).

\section{PAR TEMPS DE NEIGE}

Il est évident que la neige occupe moins de place que la pluie ou le vent. Mais, au début du $\mathrm{XX}^{\mathrm{e}}$ siècle, la Belgique connaît encore des hivers bien froids, avec des gels et de la neige en abondance. Paul Willems l'évoque en corollaire du vent :

Mais la sensation la plus exquise est d'être réveillé au milieu de la nuit par le silence. Un silence étrange. On a l'impression que ce silence-là, on le touche de la pulpe des doigts. L'air est comme emmitouflé de fourrures. On ne sait pas pourquoi, mais on est heureux. On devine qu'un grand ange, l'ange gardien du vent, s'est posé dans un arbre, immobile, silencieux pour ne pas perturber le miracle inouï qui s'accomplit. On ouvre les yeux. Tiens ! Quelle est cette lumière diffuse ? Est-ce la lumière du silence ? Et alors un élan de joie vous réveille tout à fait. Je sais ce qui s'est passé avant d'avoir nommé l'événement. Le mot vient avec un léger retard. Il est devenu inutile car je sais déjà ce qui est arrivé. Tous mes sens en sont alertés. Je murmure en souriant : « Il neige » (Willems, 1998, p. 15) ${ }^{21}$.

Notre hypothèse de base était que la neige apporte une délivrance en couvrant par une couche blanche et innocente tout ce qu'on veut repousser et ne pas voir en face. La seule pièce qui y recourt dans son univers diégétique est Warna ou le poids de la neige $e^{22}$, c'est-à-dire, une de ses pièces les plus tragiques.

La neige y apparaît dès la première scène et est omniprésente dans le discours des personnages. Au point que l'on pourrait croire qu'elle n'arrête pas de tomber tout au long de la pièce. Rien de tel. Toutefois, le lecteur/spectateur ne la voit arriver qu'en quatrième acte. Mais avant sa première apparition, les protagonistes évoquent ses multiples qualités : «[...] le chant de la neige... De joie calme. D’une joie toute blanche, sereine. [...] on dirait que cette neige abolit la souffrance. Neige. Pureté. On y chercherait en vain l'empreinte d'une seule larme. [...] Ce sont les moments de tendresse » (WPN, p. 225) pour n'en citer que quelques unes. Les personnages l'accueillent à bras ouverts. Anita qui s'exclame à deux reprises : "Regarde, la neige vient sur mes mains... »

${ }^{21}$ C'est l'auteur qui souligne.

${ }^{22}$ Pour les références de cette pièce, nous abrégerons le titre en $W P N$, suivi de la pagination qui renvoient à l'édition de la bibliographie finale. 
(p. 274), « Regarde ! C'est beau ! Ça vit, c'est le vent blanc ! C'est la vie douce sur les mains... ça coule... ça danse... Marthe ! je crois que c'est le bonheur !» (p. 294).

Néanmoins, cette même neige devient de plus en plus lourde à supporter pour d'autres personnages qui en souffrent et attendent le printemps. Le contraste est bien clair dans la réplique de Visius : « Le ciel n'a jamais été aussi noir, ni la neige aussi blanche » (p. 293). Ginette Michaux avance même que, dans la seconde partie de la pièce, « le poids de la neige deviendra celui de l'amour qui a grossi, à l'image d'Ernevelde $[\ldots] »$ (Michaux, 1988, p. 134), donc quelque chose de très pesant.

Tant que la neige est stable, solide, la réalité semble être fixée : «[...] la gelée, qui viendra tout figer» (WPN, p. 298), «[...] je marchais dans un cauchemar gelé » (p. 312), mais dès que la neige commence à fondre, les forces qui régissent les personnages changent et deviennent plus liquides ${ }^{23}$. De cette façon, la configuration des liens actantiels ${ }^{24}$ est bouleversée et l'intrigue peut tendre vers la fin et se dénouer. L'exergue du drame atteint une portée métaphysique, ce que les critiques soulignent dans leurs travaux. Et nous ne pouvons qu'approuver l'opinion de Thérèse Deman qui constate : «Tout nous laisse croire, dans cette finale, que les personnages de Warna ont atteint quelque chose de surnaturel : la lumière étincelante, la neige devenue légère et pure, la statue de la neige resplendissante, sont autant de signes de la divinité retrouvée »(Deman, 1988, p. 124).

\section{PAR TEMPS DE SOLEIL}

Pour ce qui est du soleil, il serait plus judicieux de parler des schèmes de transitivité entre plusieurs pièces de l'auteur de La Plage aux anguilles car nous n'avons pas constaté de dominante solaire dans aucun texte soumis à l'analyse. De surcroit, notre hypothèse de recherche concernant le rôle du soleil qui apporte aux personnages le soulagement et les forces vitales trouverait sa confirmation dans la plupart des textes étudiés.

Ainsi, dans Il pleut dans ma maison, la plus pluvieuse de toutes les pièces, Germaine évoque un agréable souvenir de jeunesse : « Nous avons grimpé dans le grand tilleul, tout vert, tout jaune, tout poisseux de fleurs. Quel parfum ! Oui ! Un parfum jaune, jaune... et le soeil. Autour de nous, l'été, c'est alors qu'il a dit : «le temps s'arrête » $[\ldots] »(P M M$, p. 90). La pièce se termine par un beau temps : «Le jour s'est levé. Un beau rayon de soleil éclaire la pièce » (PMM, p. 153).

L'univers diégétique de Warna est original et diffère des autres pièces de l'auteur belge. L'intrigue est située dans un château dont l'accès est très limitée ${ }^{25}$. Tous les évé-

${ }^{23}$ Dans le sens que Zygmunt Bauman attribue à ce terme.

${ }^{24}$ Sur les principes de modèle actantiel au théâtre, voir Ubersfeld, 1996, pp. 43-88.

${ }^{25}$ Le château est entouré d'eau comme s'il se trouvait sur une île. Une autre pièce de Willems est dotée d'un même emplacement, à savoir Elle disait dormir pour mourir. Par contre, elle est la seule à se passer en hiver, par temps de neige. 
nements se passent en hiver. Cette saison est souvent nuageuse et voit rarement les rayons de soleil ce qui trouve un écho dans la pièce. En effet, le soleil n'apparaît que deux fois. La première, quand Warna dicte à Visius sa lettre quotidienne adressée au chevalier Ernevelde : « ...L'amour, comme le soleil, m’éclaire et m'apaise... Le soleil, comme l'amour, éclaire les étangs et les apaise... » (WPN, p. 246); la deuxième, à l'aube, au lendemain d'une attaque attendue des ennemis : « Le soleil ! Le danger est passé. [...] Entre la lune et le soleil, il y a le temps de la mort» (WPN, p. 270) constate Ernevelde.

Un autre exemple se trouve dans La Ville à voile où Anne-Marie s'écrie : «Quel joli soleil ! Le trottoir est plein de jonquilles ${ }^{26} »(\mathrm{VV}$, p. 94). Son amoureux, Dile, l'évoque aussi : « Un peu de soleil marin que j'ai volé pour cet hiver » (VV, p. 137). Il rêve d'une vie riche aux côtés de la jeune fille : « Nous marcherons dans la rue du côté où il y a du soleil. Tu as des violettes dans les yeux, tu ris en te regardant dans les vitrines et jamais nous ne deviendrons vieux » (VV, p. 105).

Dans la partie introductive de cet article, nous avons évoqué le caractère foncier de la poétique willemsienne. Tony, un jeune garçon de dix-sept ans, semble un vrai porte-parole de l'auteur belge. On peut trouver dans Off et la lune une réplique significative de Simone : « Tony croit que l'influence des éléments est très importante.. [...] Il dit qu'un enfant est comme une plante et que sa mère est comme la terre où pousse la plante... [...] Il te faut du soleil, du vent et même de la pluie... La pluie sur les mains et la figure, c'est doux... » $(O L$, p. 90).

\section{EN GUISE DE CONCLUSION}

« La force du théâtre n'est pas d'imiter, mais de créer une autre réalité plus forte que celle de la vie quotidienne. Et c'est une force immense dont dispose le théâtre. Il crée le mythe sans lequel nous ne pourrions vivre » (Willems, 1998, p. 101)

En parlant de l'œuvre de sa mère Marie Gevers, Willems constate que « chaque mot qu'on y lit évoque à la fois le temps qui passe et le temps qu'il fait »(Willems, 1989, p. 7 ; l'auteur souligne). Il en est de même dans ses propres créations, par exemple Madeleine (on ne sait pas si c'est la nièce ou la tante...) s'écrie devant le fantôme de Herman : «Viens, le temps est là. Quittons les vivants » (PMM, p. 151).

Après avoir étudié les « schèmes de transitivité » ou « l'unité de la palette " ${ }^{27}$ selon la terminologie willemsienne, nous pouvons constater la présence des éléments naturels liés au temps et aux saisons dans la plupart de ses œuvres dramatiques. Nous

${ }^{26}$ Les jonquilles apparaissent aussi dans Il pleut dans ma maison où Bulle constate : «Quelle différence entre une femme, une jonquille et un jour de pluie... » (PMM, p. 133).

${ }^{27}$ Nous nous sommes concentrée uniquement sur ses composantes liées au temps et aux saisons, conformément à la thématique proposée pour le présent volume. Il existe, bien évidemment, d'autres motifs - tout aussi intéressants - qui méritent une étude à part. 
avons repéré les composantes "météorologiques » essentielles qui forment un ensemble cohérent et bien déterminé dans l'univers magique de Paul Willems. Nous pouvons confirmer aussi nos hypothèses formulées au début de cette étude sur le rôle apaisant et délivrant de la pluie, signe d'optimisme et d'espérance pour le soleil, avertissement par rapport au vent. Le cas de la neige s'avère plus compliqué car sa présence est ambiguë : d'une part, elle couvre tout par sa nappe blanche et « abolit toute souffrance » (Deman, 1988, p. 122), mais d'autre part, le contraste entre sa blancheur, sa pureté et les couleurs « sales » du monde devient plus fort et plus terrifiant. 


\section{BIBLIOGRAPHIE}

Deman, T. (1988). Warna ou le poids de la neige : un rêve d'ailleurs. Textyles, 5, 117-125. URL : http:// textyles.revues.org/1714; DOI : 10.4000/textyles.1714.

Gasparro, R. (2002). Pirandello et Willems : le sourire dans un miroire sombre. In F. van de Keckhove (Ed.), Paul Willems, l'enchanteur. New York : Peter Lang Publishing.

Gasparro, R. (2014). Oltre il giardino. Paul Willems tra narrativa e teatro. Rome: Bulzoni Editore.

Loriau, H.-F., Pantkowska, A. (2001). Paul Willems : le dernier écrivain belge ? Studia Romanica Posnaniensia, 28, 149-162.

Meurée, Ch. (2018). Introduction à Euvres 3 de Paul Willems. Bruxelles : Archives et Musée de la Littérature.

Michaux, G. (1988). Genres et écriture dans Warna ou le poids de la neige. Textyles, 5, 127-141. URL : http://textyles.revues.org/1716; DOI : 10.4000/textyles.1716.

Pavis, p. (2009). Dictionnaire du théâtre. Paris : Armand Colin.

Quaghebeur, M. (1992). La Ville à voile, un accomplissement. In. P. Emond, H. Ronse et F. van de Keckhove (Ed.), Le Monde de Paul Willems. Textes, entretiens, études (pp. 306-319). Bruxelles : Éditions Labor.

Quaghebeur, M. (2014, décembre). Documentaire Missembourg, creuset de la création littéraire de Marie Gevers et Paul Willems. Interview de Jan Willems par Marc Quaghebeur. Version complète. Production Archives et Musée de la Littérature. Publié le 19 mai 2016, https://www.youtube.com/ watch? $\mathrm{v}=\mathrm{mYFjFzev-JE}$, consulté le 24 juillet 2020.

Quaghebeur, M. (2017). Du fantastique réel au réalisme magique ou d'une guerre mondiale à l'autre. Tout est réel ici de Paul Willems. In R. Bizek-Tatara (Ed.), Au-délà du réel. Écritures du surnaturel dans les Lettres belges francophones (pp. 15-43). Lublin : Wydawnictwo Uniwersytetu Marii Curie-Skłodowskiej.

Richard, J.-P. (1964). Onze études sur la poésie moderne. Paris : Éditions du Seuil.

Ubersfeld, A. (1996). Lire le théâtre I. Paris : Éditions Belin.

Willems, P. (1975). Marie Gevers et le jardin de Missembourg. Revue des Deux Mondes, novembre, 324 329. Document pdf disponible en ligne : https://www.revuedesdeuxmondes.fr/article-revue/mariegevers-et-le-jardin-de-missembourg/, consulté le 25 juillet 2020.

Willems, P. (1989). La Ville à voile suivi de La Vita breve. Bruxelles : Éditions Labor.

Willems, P. (1989). Le Fonds Marie Gevers et ses prolongements. Bruxelles : Académie royale de langue et de littérature françaises de Belgique. Document pdf disponible en ligne : https://www.arllfb.be/ ebibliotheque/communications/willems041189.pdf, consulté le 25 juillet 2020.

Willems, P. (1995). Théâtre (1954-1962). Off et la lune, La Plage aux anguilles, Marceline. Bruxelles : Éditions Labor et Archives et Musée de la Littérature.

Willems, P. (1998). Un arrière-pays. Rêveries sur la création littéraire ( $2^{\text {nd }}$ éd., la première de 1989). Louvain-La-Neuve : Presses Universitaires de Louvain UCL, Chaire de Poétique 3.

Willems, P. (2000). Elle disait dormir pour mourir. Bruxelles : Cahiers du Rideau 25.

Willems, P. (2004). Vers le théâtre. Écrits 1950-1992. Bruxelles : Archives et Musée de la Littérature.

Willems, P. (2018). Euvres 3 (Il pleut dans ma maison. Warna ou le poids de la neige). Bruxelles : Archives et Musée de la Littérature. 